\title{
OPTIMAL CONTROL IN DISCRETE PEST CONTROL MODELS
}

\author{
Author: \\ KATHRYN DABBS \\ University of Tennessee \\ (865)406-1971 \\ katdabbs@gmail.com
}

\author{
Supervisor: \\ Dr. Suzanne Lenhart \\ University of Tennessee \\ (865)974-4270 \\ lenhart@math.utk.edu
}

\section{Abstract}

We use discrete time models to represent the dynamics of two interacting populations, a "valuable" population and a "pest" population. We investigate optimal control in the form of decreasing the growth rate of the "pest" population with the goal of maximizing the "valuable" population while minimizing the cost of the control. We compare different types of growth functions for the "valuable" population and their impact on the optimal control.

\section{INTRODUCTION}

The problem of invasive species has become common in much of the world, and their environmental threats and economic impacts are significant.[9] For management, invasion dynamics and cost of control actions are key issues. A recent review paper by EpanchinNeill and Hastings, [4] synthesizes modeling approaches to optimal management and the inclusion of various economic factors. The book by Hawkins and Cornell [5] illustrates how modeling tools can make biological control more predictable. When developing plans for controlling the invasive or "pest" population, optimal control theory can be applied to appropriate models $[7,8]$.

In this work, we use discrete time models to represent the dynamics of two interacting populations, a "valuable" population and a "pest" population. We investigate optimal control in the form of decreasing the growth rate of the "pest" population with the goal of maximizing the "valuable" population while minimizing the cost of the control. We compare different types of growth functions for the "valuable" population and their impact on the optimal control. The growth functions we compare include the logistic growth function, the Beverton-Holt growth function, and the Ricker spawner-recruit curve. See the book by Kot [6] for further discussion of such growth functions in discrete time models.

This paper is organized as follows: In section 3 we give a background of optimal control applied to discrete time models. In sections 4, 5, and 6 we introduce our models, formulate our optimal control problems, and compare the optimal control results for the logistic, Beverton-Holt, and Ricker growth functions, respectively. In section 7 we present a comparison of the optimal control of the logistic and Ricker growth functions and the optimal control of the logistic and Beverton-Holt growth functions. 


\section{Background of Optimal Control of Discrete Time Models}

Given a control $u=\left(u_{0}, u_{1}, \ldots, u_{T-1}\right)$ and an initial state $x_{0}$, the state equation is given by the difference equation

$$
x_{k+1}=g\left(x_{k}, u_{k}, k\right) \text { for } k=0,1,2, \ldots, T-1 .
$$

Note that the state, $x=\left(x_{0}, x_{1}, \ldots, x_{T}\right)$, has one more component than the control.

Our goal is to maximize or minimize the following objective function:

$$
J(u)=\phi\left(x_{T}\right)+\sum_{k=0}^{T-1} f\left(x_{k}, u_{k}, k\right) .
$$

Necessary conditions, which must be satisfied by the control and the corresponding state, can be derived using a generalization of Pontryagin's Maximum Principle [8], similar to the case of ordinary differential equations. A detailed derivation of the necessary conditions is given by Lenhart and Workman [7].

The key idea is to construct a function called the Hamiltonian by introducing the adjoint function to attach the difference equation to the objective function. This principle converts the problem of finding the control to optimize the objective function subject to the state difference equation with initial condition to finding the control to optimize the Hamiltonian pointwise with respect to the control.

Now we can define the Hamiltonian at each time step $k$,

$$
H_{k}=f\left(x_{k}, u_{k}, k\right)+\lambda_{k+1} g\left(x_{k}, u_{k}, k\right), \quad \text { for } k=0,1, \ldots, T-1,
$$

where $\lambda=\left(\lambda_{0}, \lambda_{1}, \ldots, \lambda_{T}\right)$ is the adjoint function.

Note that the indexing for the adjoint function is one step ahead of the indexing for the other terms. The necessary condition states that the Hamiltonian is maximized at each step with respect to the control $u_{k}$ at the optimal control $u_{k}^{*}$. The adjoint equations and corresponding final time conditions or transversality conditions are also given. If there are no constraints on the control, the necessary conditions are

$$
\begin{aligned}
\lambda_{k} & =\frac{\partial H_{k}}{\partial x_{k}} \\
\lambda_{T} & =\phi^{\prime}\left(x_{T}^{*}\right) \\
\frac{\partial H_{k}}{\partial u_{k}} & =0 \text { at } u^{*} .
\end{aligned}
$$

Note that the adjoint function must satisfy final time conditions while the state function must satisfy initial time conditions. Suppose that the controls are bounded, which is often the case in biological examples. Suppose $a \leq u_{k} \leq b$ for each $k$. Then these bounds need to be imposed after solving the optimality equation

$$
\frac{\partial H_{k}}{\partial u_{k}}=0 \text { at } u^{*}
$$

for each component of the control at each time step. This idea can be easily generalized to a system with multiple states. In the case of multiple states, there is a corresponding 
adjoint function for each state function.

\section{Optimal Control with the Logistic Growth Function}

The valuable population and pest populations are represented by

$$
x=\left(x_{0}, x_{1}, \ldots, x_{T}\right)
$$

and

$$
y=\left(y_{0}, y_{1}, \ldots, y_{T}\right),
$$

respectively, where the subscripts represent the time steps.

The model equation for the valuable population is:

$$
x_{k+1}=x_{k}+c x_{k}\left(1-\frac{x_{k}}{K}\right)-r x_{k} y_{k} \quad \text { for } k=1,2, \ldots, T-1,
$$

where $x_{k}$ is the valuable population at the previous time step $k, x_{k+1}$ is the valuable population at the next time step $k+1, \mathrm{r}$ is a population interaction constant which measures the efficiency of the pest population, $\mathrm{c}$ is the intrinsic growth rate of the valuable population, and $\mathrm{K}$ is the carrying capacity.

The dynamics of the pest population are simpler:

$$
y_{k+1}=d y_{k}+x_{k} y_{k}
$$

where $y_{k}$ is the pest population at the previous time step $k, y_{k+1}$ is the pest population at the next time step $k+1$, and $\mathrm{d}$ is the intrinsic growth rate of the pest population. Note that $x_{0}$ and $y_{0}$ are assumed to be known.

4.1. Application of the control. Let $u=\left(u_{0}, u_{1}, \cdots, u_{T-1}\right)$ be the control, which we apply to decrease the growth of the pest population, by decreasing the growth rate of the $y$ population at each time step. The control satisfies:

$$
U=\left\{\left(u_{0}, \ldots, u_{T-1}\right) \in \mathbf{R}^{T} \mid 0 \leq u_{k} \leq M \leq 1\right\} .
$$

Our objective function, representing our goal, is:

$$
J(u)=\sum_{k=0}^{T-1}-B_{2} y_{k}+\frac{1}{2}\left[x_{k}-B_{1} u_{k}^{2}\right]
$$

over $u \in U$, subject to

$$
\begin{gathered}
x_{k+1}=x_{k}+c x_{k}\left(1-\frac{x_{k}}{K}\right)-r x_{k} y_{k} \quad \text { for } k=0,1, \cdots, T-1, \text { given } x_{0}, \\
y_{k+1}=d y_{k}+x_{k} y_{k}-u_{k} y_{k} \quad \text { for } k=0,1, \cdots, T-1, \text { given } y_{0} .
\end{gathered}
$$

The goal is maximizing the valuable population while minimizing the pest population and the cost of the control. Note that the terms in the objective function which correspond to the pest population and the control are negative, while the term which corresponds to the 
valuable population is positive. Thus, by maximizing $J(u)$ over $u \in U$, we can achieve the desired minimization of the pest population and cost of the control.

Theorem 1 There exists an optimal control $u^{*} \in U$, with corresponding states from (5)-(6) such that $J\left(u^{*}\right)=\max _{u \in U} J(u)$.

Proof. Since the coefficients of the state equations are bounded and there are a finite number of time steps, $x=\left(x_{0}, x_{1}, \ldots, x_{T}\right)$ and $y=\left(y_{0}, y_{1}, \ldots, y_{T}\right)$ are uniformly bounded for all $u \in U$. Thus $J(u)$ is uniformly bounded for all $u$ in the control set $U$. Since $J(u)$ is bounded, $\sup _{u \in U} J(u)$ is finite, and there exists a sequence $u^{j} \in U$ such that $\lim _{j \rightarrow \infty} J\left(u^{j}\right)=\sup _{u \in U} J(u)$ and corresponding sequences of states $x^{j}$ and $y^{j}$. Since there is a finite number of uniformly bounded sequences, there exists $u^{*} \in U$ and $x^{*}, y^{*} \in \mathbf{R}^{T+1}$ such that on a subsequence, $u^{j} \rightarrow u^{*}, x^{j} \rightarrow x^{*}$, and $y^{j} \rightarrow y^{*}$. Then, from the structure of the state equations and the objective functional $J(u), u^{*}$ is an optimal control with corresponding states $x^{*}$ and $y^{*}$. Therefore $\sup _{u \in U} J(u)$ is achieved.

Applying the discrete version of Pontryagin's Maximum Principle[7], we form the Hamiltonian:

$$
H_{k}=-B_{2} y_{k}+\frac{1}{2} x_{k}-\frac{B_{1}}{2} u_{k}^{2}+\lambda_{x, k+1}\left(x_{k}+c x_{k}\left(1-\frac{x_{k}}{K}\right)-r x_{k} y_{k}\right)+\lambda_{y, k+1}\left(d y_{k}+x_{k} y_{k}-u_{k} y_{k}\right),
$$

which is used to derive the necessary conditions in the next theorem.

Theorem 2 Given an optimal control $u^{*} \in U$ and corresponding states $x^{*}$ and $y^{*}$ from (5)-(6), there exist adjoint functions $\lambda_{x}$ and $\lambda_{y}$ satisfying

$$
\begin{gathered}
\lambda_{x, k}=\frac{1}{2}+\lambda_{x, k+1}\left(1+c-\frac{2 c x_{k}^{*}}{K}-r y_{k}^{*}\right)+\lambda_{y, k+1} y_{k}^{*} \\
\lambda_{y, k}=-B_{2}-\lambda_{x, k+1} r x_{k}^{*}+\lambda_{y, k+1}\left(d+x_{k}^{*}-u_{k}^{*}\right) \\
\lambda_{x, T}=\lambda_{y, T}=0 .
\end{gathered}
$$

Furthermore, $u^{*}$ satisfies

$$
u_{k}^{*}=\min \left\{\max \left\{\frac{-\lambda_{y, k+1} y_{k}^{*}}{B_{1}}, 0\right\}, 1\right\} .
$$

Proof. Using the discrete version of Pontryagin's Maximum Principle [7],

$$
\begin{gathered}
\lambda_{x, k}=\frac{\partial H_{k}}{\partial x_{k}}=\frac{1}{2}+\lambda_{x, k+1}\left(1+c-\frac{2 c x_{k}^{*}}{K}-r y_{k}^{*}\right)+\lambda_{y, k+1} y_{k}^{*} . \\
\lambda_{y, k}=\frac{\partial H_{k}}{\partial y_{k}}=-B_{2}-\lambda_{x, k+1} r x_{k}^{*}+\lambda_{y, k+1}\left(d+x_{k}^{*}-u_{k}^{*}\right)
\end{gathered}
$$

Additionally, the adjoint final conditions, which satisfy $\lambda_{x, T}=\phi^{\prime}\left(x_{T}^{*}\right)$, are

$$
\lambda_{x, T}=\lambda_{y, T}=0 .
$$

Using

$$
\frac{\partial H_{k}}{\partial u_{k}}=-B_{1} u_{k}-\lambda_{y, k+1} y_{k}
$$


and $\frac{\partial H_{k}}{\partial u_{k}}=0$ at $u^{*}$ on the interior of the control set, we have the control characterization,

$$
u_{k}^{*}=\min \left\{\max \left\{\frac{-\lambda_{y, k+1} y_{k}^{*}}{B_{1}}, 0\right\}, 1\right\} \text {. }
$$

The optimality system for our problem consists of the state equations (5), (6) with initial conditions and adjoint equations (8), (9) with final time conditions (10) and with the optimal control characterization (11).

TABLE 1. Summary of Logistic Growth Parameters

\begin{tabular}{|c|c|c|}
\hline Parameter & Description & Value \\
\hline \hline $\mathrm{T}$ & number of time steps & 15 \\
$x_{0}$ & initial valuable population & 0.5 \\
$y_{0}$ & initial pest population & 1 \\
$\mathrm{r}$ & population interaction constant & $0.01,0.1,1,10$ \\
$\mathrm{~K}$ & carrying capacity & $0.01,0.1,1,10$ \\
$\mathrm{c}$ & intrinsic growth rate of valuable population & $0.01,0.1,1,10$ \\
$\mathrm{~d}$ & intrinsic growth rate of pest population & $0.01,0.1,1,10$ \\
$B_{2}$ & balancing coefficient & $0.01,0.1,1,10$ \\
$B_{1}$ & cost coefficient & $0.01,0.1,1,10$ \\
\hline
\end{tabular}

4.2. Numerical Results. For various parameters shown in Table 1, we present the results of solving the optimality system numerically. An iterative method is used to solve the optimality system. Starting with an initial guess for the control, we solve the state system forward and then the adjoint system backward. The control is updated using the optimal control characterization. We continue until successive iterates are close.

For the application of the control to the logistic growth function, we varied one parameter in (5) and (6) at a time over the values $0.01,0.1,1$, and 10, as described in Table 1, while keeping all other parameters in (5) and (6) constant at 1 . The initial values for the pest population and the valuable population are 1.0 and 0.5 respectively.

Figure 1 gives an example of the growth of the valuable population and the pest population without the application of the control and with the application of the optimal control, $\mathrm{u}$. Without the control, the pest population increases, killing off the valuable population. Then, when the valuable population has decreased to 0 , the pest population remains constant with a growth rate of $d=1.0$. In contrast, with the application of the optimal control, the growth rate of the pest population is decreased, allowing the valuable population to increase until approaching the carrying capacity, $K=1.0$.

Figure 2 also shows the effect of the control in decreasing the growth rate of the pest population. With a growth rate of $c=0.01$, the valuable population is removed by the pest population in one time step, when the control is not applied. The pest population then maintains a population of about 1.5 with an intrinsic growth rate of $d=1.0$. When 

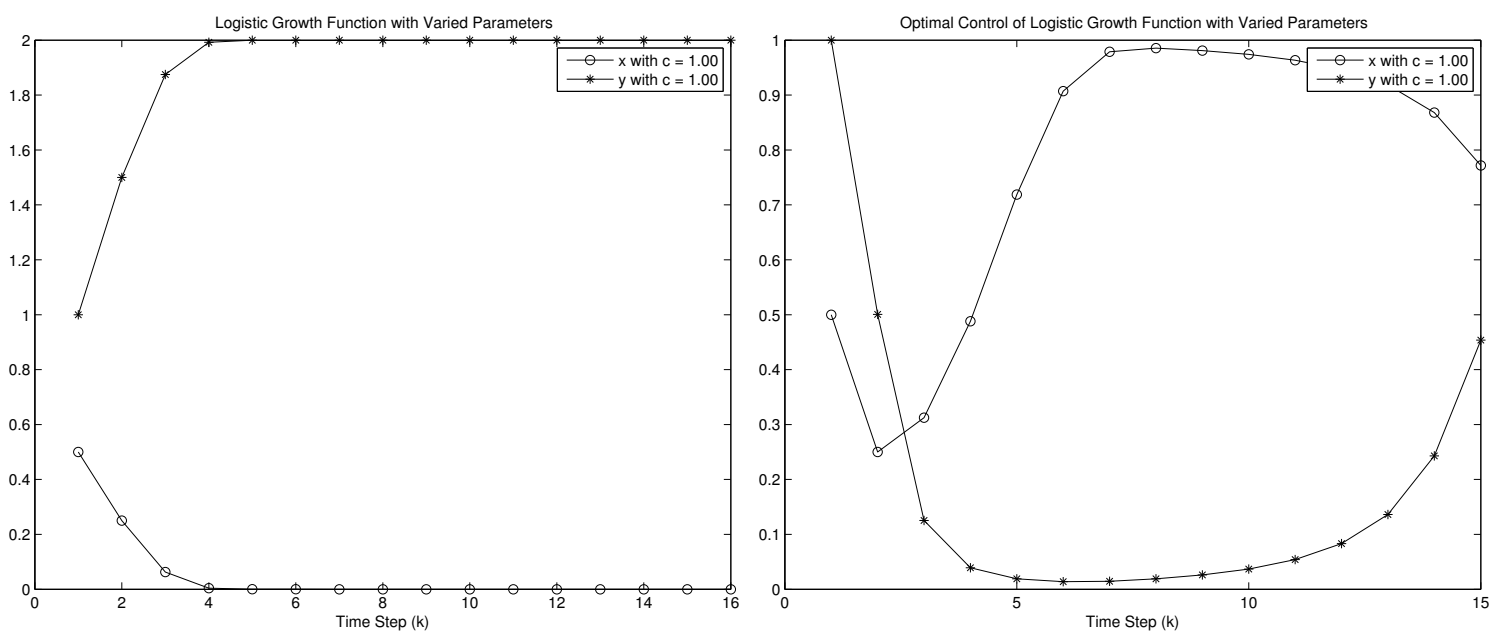

(a) Valuable population and pest population without (b) Valuable population and pest population with opcontrol timal control

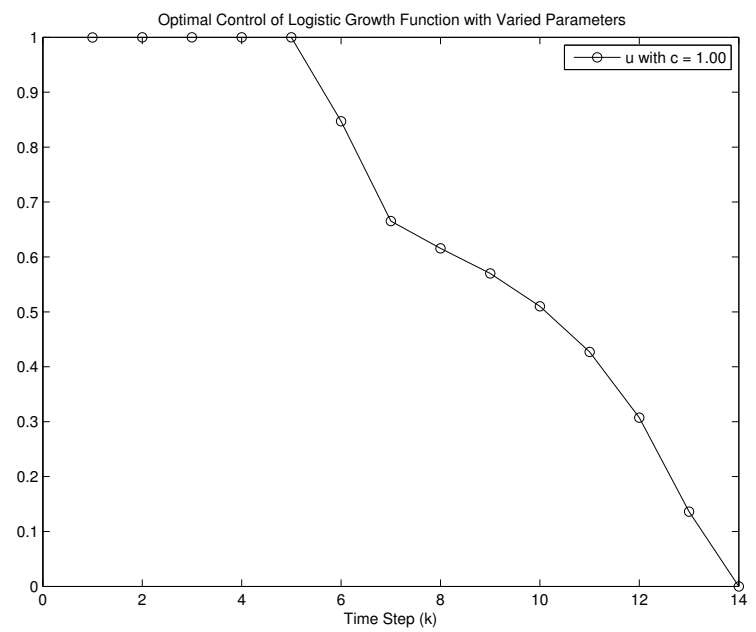

(c) Optimal control, u

FiguRE 1. Logistic growth functions with $c=d=K=r=B_{1}=B_{2}=1.0$

the optimal control is applied, the growth of the valuable population is not affected, but the growth rate of the pest population is decreased. In this case, since the growth rate of the valuable population is less than the population interaction constant $r$, the valuable population still dies out after the first time step, showing the same trend as can be seen in the system without the control. The effect of the control can be seen, however, in the growth of the pest population. The application of the control causes the pest population to decrease to 0 after the second time step. 

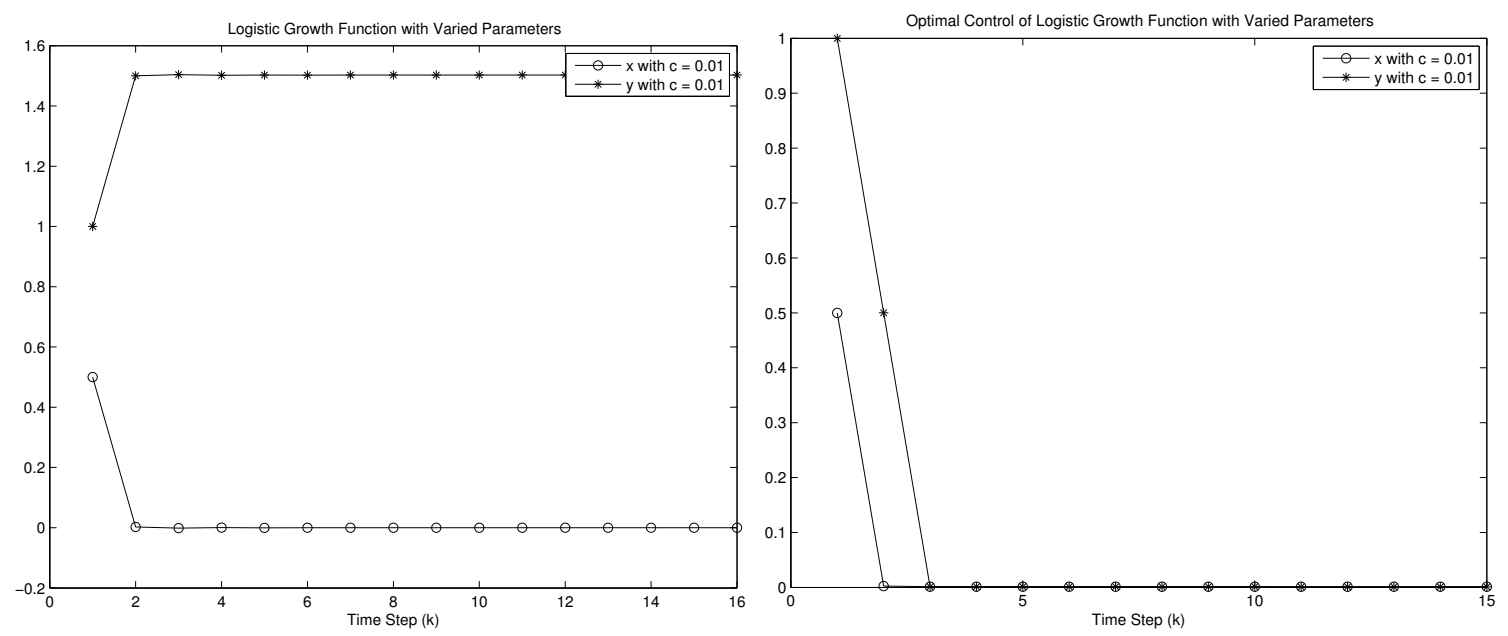

(a) Valuable population and pest population without

(b) Valuable population and pest population with opcontrol timal control

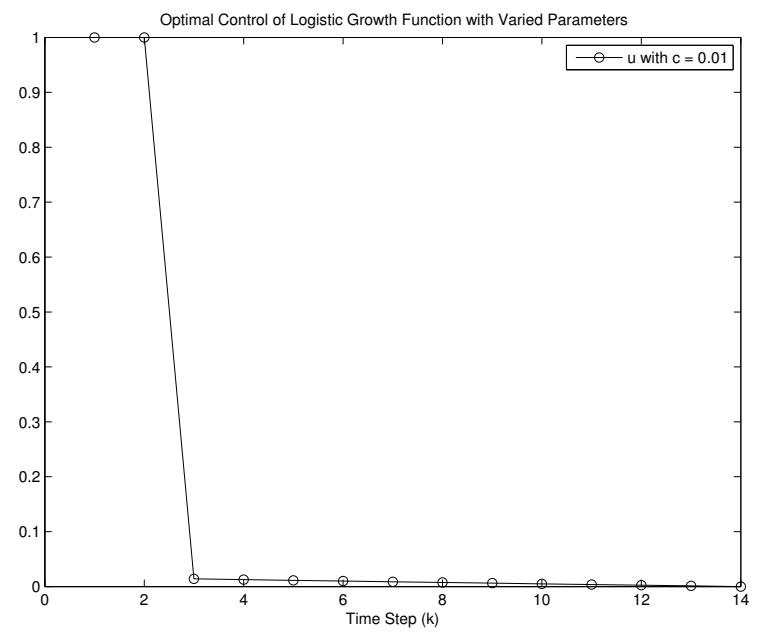

(c) Optimal control, u

Figure 2. Logistic growth functions with $c=0.01$ and $d=K=r=B_{1}=$ $B_{2}=1.0$

Figure 3 compares the optimal control, $\mathrm{u}$, as $\mathrm{d}$, the intrinsic growth rate of the pest population is varied. For $d=0.01$ and $d=0.10$ the optimal control is the same. In addition, for $d=1.0$ and $d=10$ the control is the same. This figure shows that as the pest population increases from $d=0.01$ and $d=0.10$ to $d=1.0$ and $d=10$, the optimal control increases over all time steps. This result is expected because more control needs to be applied to decrease a pest population with a higher intrinsic growth rate. 


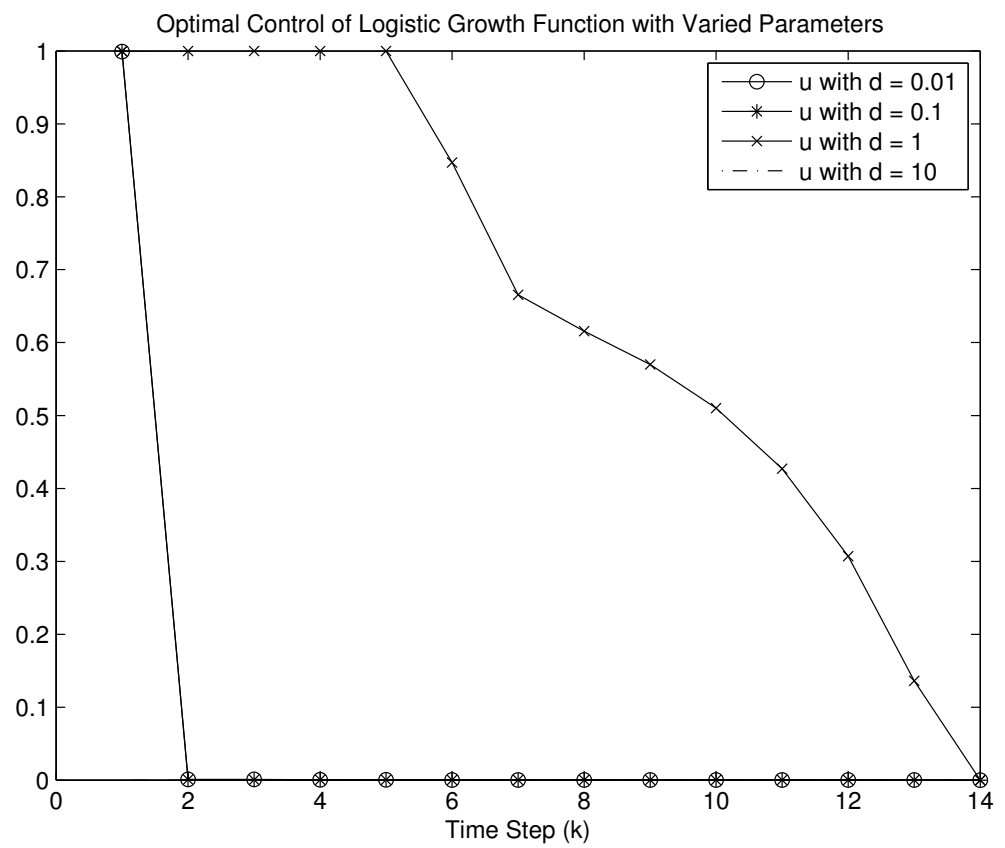

FiguRE 3. Optimal control of logistic growth function as d is varied

The behavior of the optimal control as $\mathrm{r}$, the population interaction constant, showed unexpected results. As seen in Figure 4, as the value of $\mathrm{r}$ increases, which corresponds to a more efficient pest population, the value of the control applied decreases. This may be explained by comparing the value of $r$, which takes away from the valuable population, to the value of $\mathrm{c}$ which adds to the valuable population. In this example, $\mathrm{c}$ is held constant at 1.0 while $\mathrm{r}$ is varied over $0.01,0.10,1.0,10$. With $r=10$, the valuable population is able to sustain itself with a growth rate of $c=1.0$. Without the contribution of interacting with the valuable population, the growth of the pest population is maintained only by the contribution of the intrinsic growth rate of the pest population, $d=1.0$. With $r=0.01$, on the other hand, the valuable population is able to sustain itself for more time steps with a growth rate of $c=1.0$ against the attack of the pest population. The growth of the pest population then depends on both its intrinsic growth rate and the contribution of interacting with the valuable population. Considering these two cases, it seems reasonable that more control would need to be applied with $r=0.01$ to decrease the growth of the pest population than with $r=10$. If the valuable population were more easily sustained (for example, if it had a higher intrinsic growth rate) the optimal control may show different trends. 


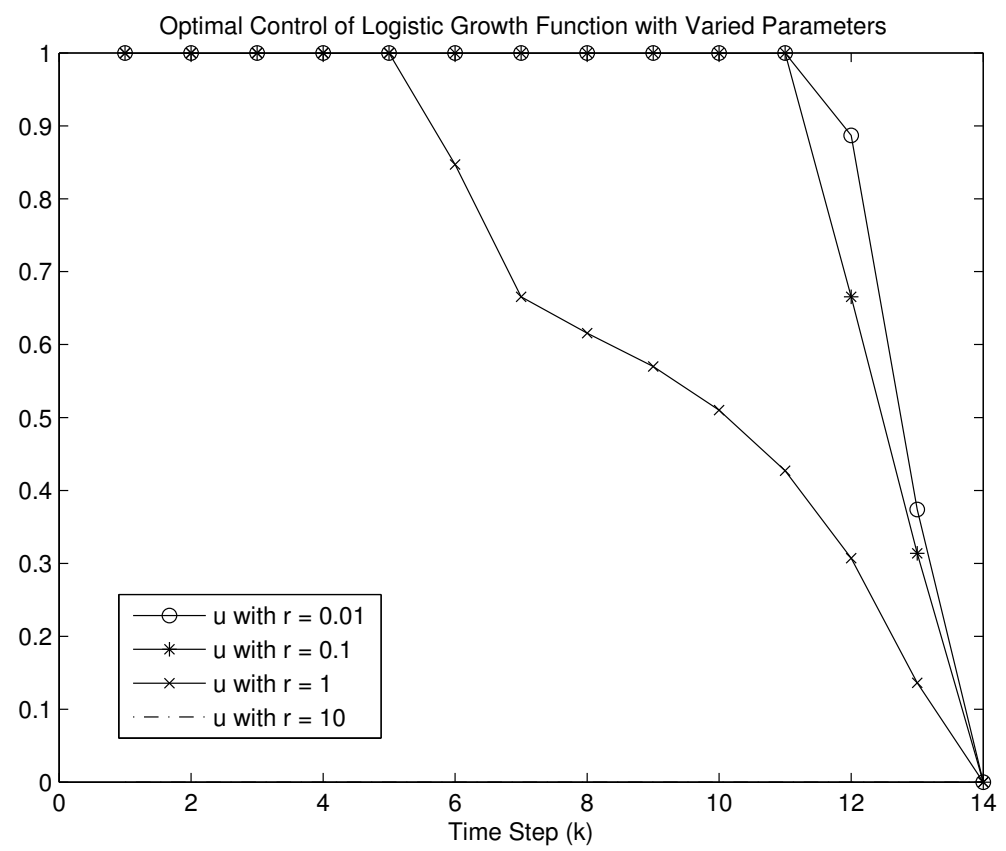

Figure 4. Optimal control of logistic growth function as $\mathrm{r}$ is varied

\section{Optimal Control with the Beverton-Holt Growth Function}

Now we consider a model with a Beverton-Holt growth function,

$$
x_{k+1}=x_{k}+\frac{R x_{k}}{1+\frac{R-1}{K} x_{k}}-r x_{k} y_{k},
$$

where $x_{k}$ is the valuable population at the previous time step $\mathrm{k}, x_{k+1}$ is the valuable population at the next time step $k+1, \mathrm{r}$ is a population interaction constant which measures the efficiency of the pest population, $\mathrm{K}$ is the carrying capacity, and $\mathrm{R}$ is the intrinsic growth rate of the valuable population. Again we choose for the pest population,

$$
y_{k+1}=d y_{k}+x_{k} y_{k},
$$

where $y_{k}$ is the pest population at the previous time step $k, y_{k+1}$ is the pest population at the next time step $k+1$, and $\mathrm{d}$ is the intrinsic growth rate of the pest population. The initial conditions, $x_{0}$ and $y_{0}$, are assumed to be known.

5.1. Application of the Control. As in the optimal control of the logistic growth function, let $u=\left(u_{0}, u_{1}, \cdots, u_{T-1}\right)$ be the control, satisfying (3), which we apply to decrease 
the growth of the pest population. Our objective functi, representing our goal, is:

$$
J(u)=\sum_{k=0}^{T-1}-B_{2} y_{k}+\frac{1}{2}\left[x_{k}-B_{1} u_{k}^{2}\right]
$$

over $u \in U$, subject to

$$
\begin{gathered}
x_{k+1}=x_{k}+\frac{R x_{k}}{1+\frac{R-1}{K} x_{k}}-r x_{k} y_{k} \quad \text { for } k=0,1, \cdots, T-1, \text { given } x_{0}, \\
y_{k+1}=d y_{k}+x_{k} y_{k}-u_{k} y_{k} \quad \text { for } k=0,1, \cdots, T-1, \text { given } y_{0} .
\end{gathered}
$$

Similar to Theorem 1 we have,

Theorem 3 There exists an optimal control $u^{*}$ in $U$ and corresponding states $x^{*}$ and $y^{*}$ from (15)-(16) such that $J\left(u^{*}\right)=\max _{u \in U} J(u)$.

Applying the discrete version of Pontryagin's Maximum Principle[7], we form the Hamiltonian:

$(17)$

$H_{k}=-B_{2} y_{k}+\frac{1}{2} x_{k}-\frac{B_{1}}{2} u_{k}^{2}+\lambda_{x, k+1}\left(x_{k}+\frac{R x_{k}}{1+\frac{R-1}{K} x_{k}}-r x_{k} y_{k}\right)+\lambda_{y, k+1}\left(d y_{k}+x_{k} y_{k}-u_{k} y_{k}\right)$

Theorem 4 Given an optimal control $u^{*}$ and corresponding states, $x^{*}$ and $y^{*}$ from (15)-(16), there exist adjoint functions $\lambda_{x}$ and $\lambda_{y}$ satisfying

$$
\begin{gathered}
\lambda_{x, k}=\frac{1}{2}+\lambda_{x, k+1}\left(1+\frac{R\left(1+\frac{R-1}{K} x_{k}^{*}\right)-R x_{k}^{*}\left(\frac{R-1}{K}\right)}{\left(1+\frac{R-1}{K} x_{k}^{*}\right)^{2}}-r y_{k}\right)+\lambda_{y, k+1} y_{k}^{*} \\
\lambda_{y, k}=-B_{2}-\lambda_{x, k+1} r x_{k}^{*}+\lambda_{y, k+1}\left(d+x_{k}^{*}-u_{k}^{*}\right) \\
\lambda_{x, T}=\lambda_{y, T}=0 .
\end{gathered}
$$

Furthermore, $u^{*}$ satisfies

$$
u_{k}^{*}=\min \left\{\max \left\{\frac{-\lambda_{y, k+1} y_{k}^{*}}{B_{1}}, 0\right\}, 1\right\} .
$$

The optimality system for our problem consists of the state equations (15), (16), with initial conditions and adjoint equations (18), (19), with final time conditions (20), and with the optimal control characterization (21).

5.2. Numerical Results. The optimality system for the Beverton-Holt growth function was solved numerically, with the same iterative method used to solve the optimal control problem for the logistic growth function. We present the results for various parameters shown in Table 2. As with the logistic growth function, we varied one parameter from (15)-(16) over the values $0.01,0.1,1.0,10$, while keeping all other parameters in (15)-(16) constant at 1.0. The initial conditions $x_{0}$ and $y_{0}$ are 0.5 and 1.0 , respectively.

Figure 5 shows the optimal control $u$ as $B_{2}$, the balancing coefficient is varied. The coefficient $B_{2}$ influences the importance of decreasing the pest population relative to maximizing 
TABLE 2. Summary of Beverton-Holt Growth Parameters

\begin{tabular}{|c|c|c|}
\hline Parameter & Meaning & Value \\
\hline \hline $\mathrm{T}$ & number of time steps & 15 \\
$x_{0}$ & initial valuable population & 0.5 \\
$y_{0}$ & initial pest population & 1 \\
$\mathrm{r}$ & population interaction constant & $0.01,0.1,1,10$ \\
$\mathrm{R}$ & intrinsic growth rate of valuable population & $0.01,0.1,1,10$ \\
$\mathrm{~K}$ & carrying capacity & $0.01,0.1,1,10$ \\
$\mathrm{~d}$ & intrinsic growth rate of pest population & $0.01,0.1,1,10$ \\
$B_{2}$ & balancing coefficient & $0.01,0.1,1,10$ \\
$B_{1}$ & cost coefficient & $0.01,0.1,1,10$ \\
\hline
\end{tabular}

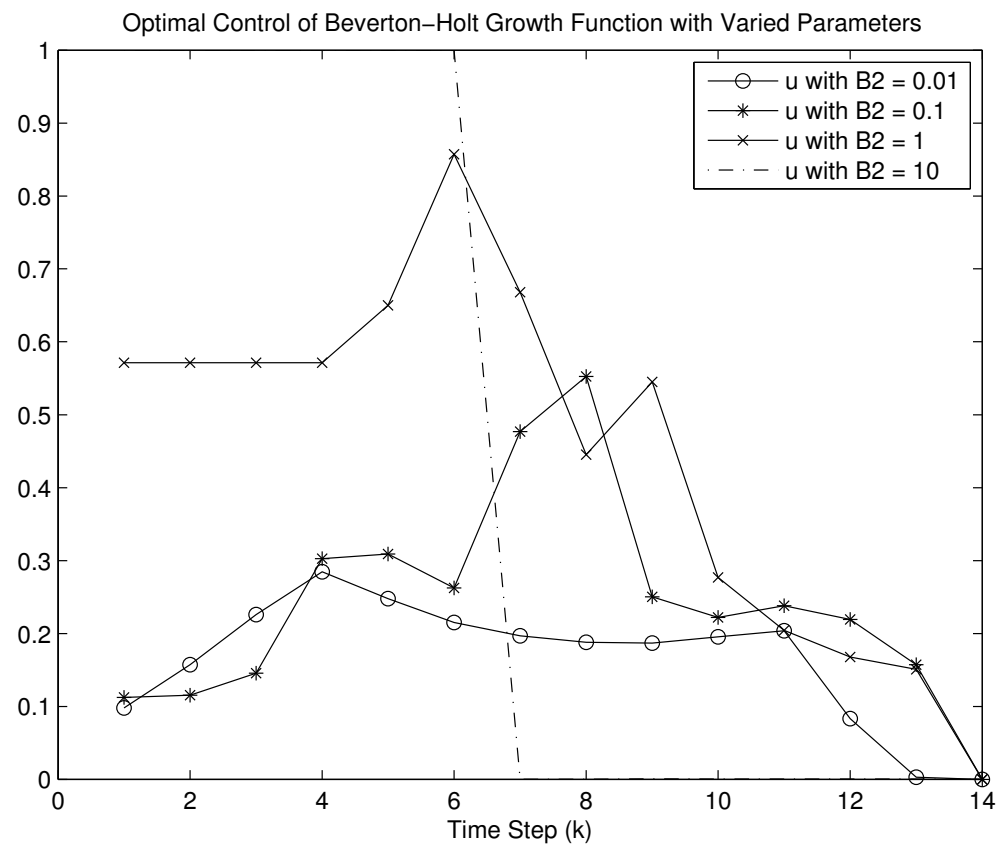

FiguRE 5. Optimal control of Beverton-Holt growth function as $B_{2}$ is varied

the valuable population and minimizing the cost of applying the control. Considering the objective function (14) shows that as $B_{2}$ decreases the importance of minimizing the pest population also decreases. This is verified by the trend shown in Figure 5 . As $B_{2}$ decreases from 1.0 to 0.1 and from 0.1 to 0.01 , the control $u$ decreases over almost all time steps. For $B_{2}=10$, the control has the highest value until it drops to around 0. Examining the 
growth of the pest and valuable populations shows that this is because the pest population dies out after the initial application of the control. Then, since minimizing the pest population is the most important goal in this case, the extinction of the pest population eliminates the need for the control.

In the Beverton-Holt growth model, $R$ represents the intrinsic growth rate of the valuable population. Figure 6 compares a system with $R=0.1$ and $d=K=r=B_{1}=B_{2}=1.0$, without the application of the control and with the application of the control. Without the control, the low intrinsic growth rate of the valuable population causes the pest population to overwhelm the valuable population, which decreases to 0 . With the application of the optimal control, the growth rate of the pest population is decreased while the growth of the valuable population is maximized. Figure 6 shows that, although the valuable population is initially lower and has a lower intrinsic growth rate than the pest population, applying the control allows the growth of the valuable population to exceed the growth of the pest population.

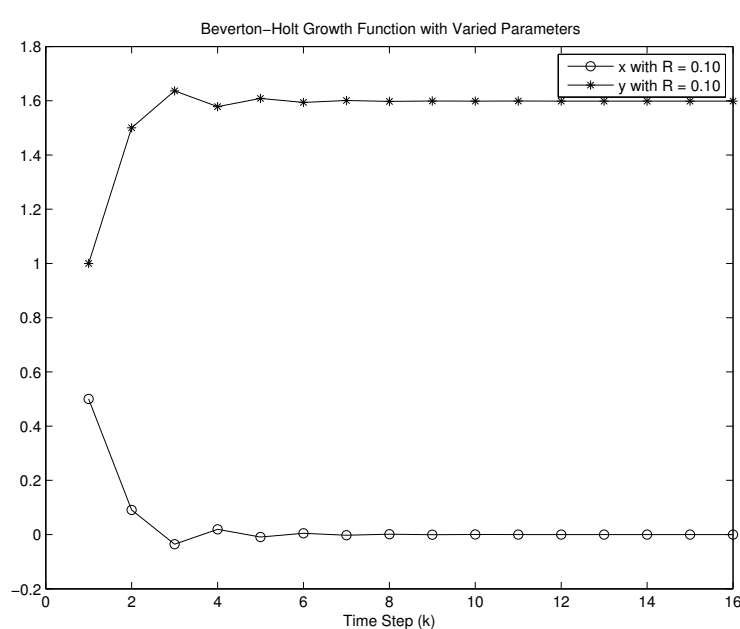

(a) Valuable population and pest population without control

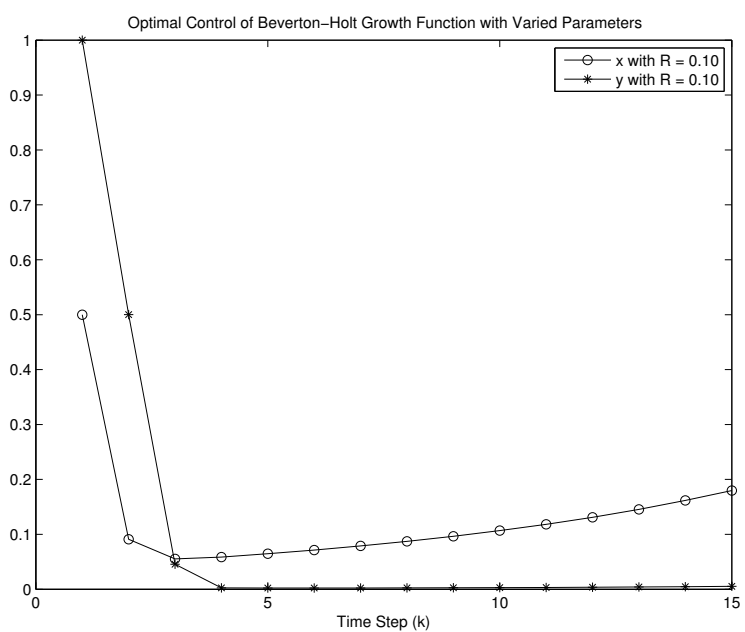

(b) Valuable population and pest population with control

Figure 6. Beverton-Holt growth functions with $R=0.1$ and $d=K=r=$ $B_{1}=B_{2}=1.0$

In contrast to the control results of the logistic growth model, in the Beverton-Holt model varying $\mathrm{K}$, the carrying capacity of the valuable population, over $0.01,0.1,1.0,10$, shows no change in the control. This is shown in Figure 7.

\section{Optimal Control with the Ricker Spawner-Recruit Curve}

For the Ricker spawner-recruit curve, the growth of the valuable population is represented by

$$
x_{k+1}=x_{k} e^{c\left[1-\frac{x_{k}}{K}\right]}-r x_{k} y_{k}
$$




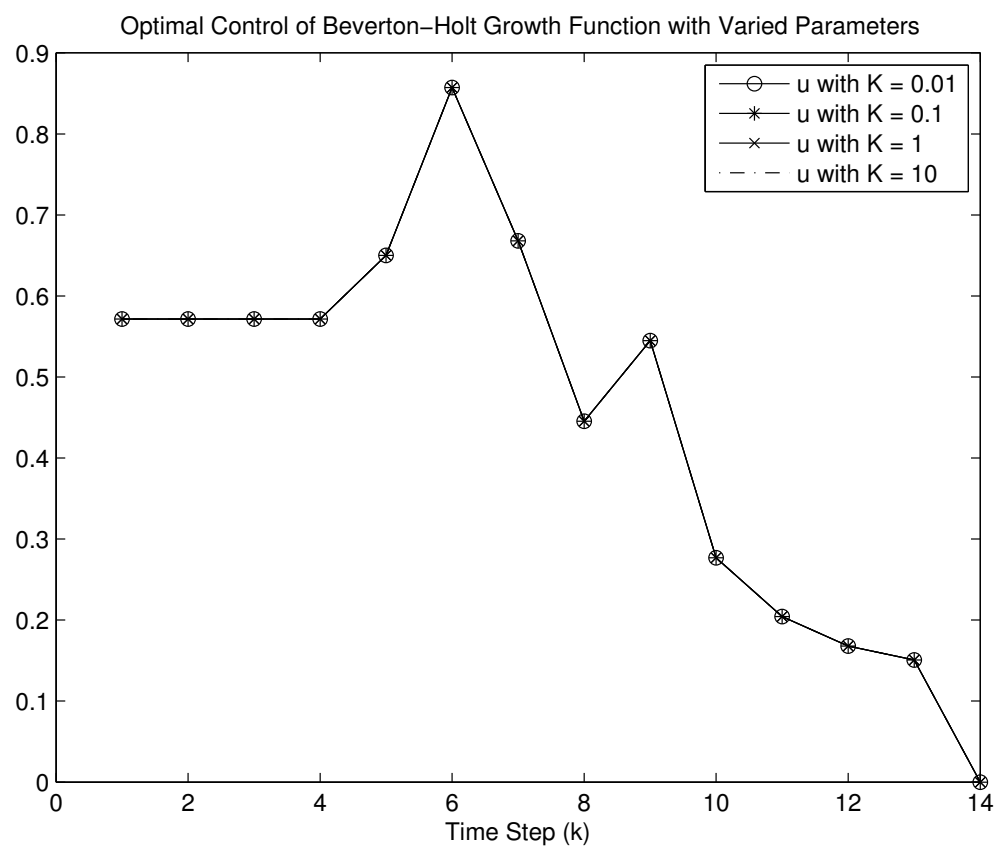

Figure 7. Optimal control of Beverton-Holt growth function as $\mathrm{K}$ is varied

where $x_{k}$ is the valuable population at the previous time step $\mathrm{k}, x_{k+1}$ is the valuable population at the next time step $k+1$, c is the intrinsic growth rate of the valuable population, $\mathrm{r}$ is a population interaction constant, and $\mathrm{K}$ is the carrying capacity of the valuable population. As in the logistic and Beverton-Holt models, the pest population is given by

$$
y_{k+1}=d y_{k}+x_{k} y_{k}
$$

where $y_{k}$ is the pest population at the previous time step $k, y_{k+1}$ is the pest population at the next time step $k+1$, and $\mathrm{d}$ is the intrinsic growth rate of the pest population. $x_{0}$ and $y_{0}$ are the initial conditions, which are assumed to be known.

6.1. Application of the Control. As in the control of the Beverton-Holt and logistic growth functions, we apply the control $u=\left(u_{1}, u_{2}, \cdots, u_{n}\right)$, satisfying (3), to decrease the growth of the pest population. The goal, which is to minimize the pest population and the cost of applying the control, while maximizing the valuable population is represented by the objective function:

$$
J(u)=\sum_{k=0}^{T}-B_{2} y_{k}+\frac{1}{2}\left[x_{k}-B_{1} u_{k}^{2}\right]
$$


over $u \in U$ subject to

$$
\begin{array}{ll}
x_{k+1}=x_{k} e^{c\left[1-\frac{x_{k}}{K}\right]}-r x_{k} y_{k} & \text { for } k=0,1, \cdots, T-1, \\
y_{k+1}=d y_{k}+x_{k} y_{k}-u_{k} y_{k} & \text { for } k=0,1, \cdots, T-1 .
\end{array}
$$

Theorem 5 There exists an optimal control $u^{*}$ in $U$ with corresponding states $x^{*}$ and $y^{*}$ from (23)-(24) such that $J\left(u^{*}\right)=\max _{u \in U} J(u)$.

Applying the discrete version of Pontryagin's Maximum Principle [7], from the objective function we form the Hamiltonian,

$$
H_{k}=-B_{2} y_{k}+\frac{1}{2} x_{k}-\frac{B_{1}}{2} u_{k}^{2}+\lambda_{x, k+1}\left(x_{k} e^{c\left[1-\frac{x_{k}}{K}\right]}-r y_{k}\right)+\lambda_{y, k+1}\left(d y_{k}+x_{k} y_{k}-u_{k} y_{k}\right) .
$$

Theorem 6 Given an optimal control $u^{*}$ and corresponding states, $x$ and $y$ from (23)-(24), there exist adjoint functions $\lambda_{x}$ and $\lambda_{y}$ satisfying

$$
\begin{gathered}
\lambda_{x, k}=\frac{1}{2}+\lambda_{x, k+1}\left(e^{c\left[1-\frac{x_{k}^{*}}{K}\right]}\left[1-\frac{c x_{k}^{*}}{K}\right]-r y_{k}^{*}\right)+\lambda_{y, k+1} y_{k}^{*} \\
\lambda_{y, k}=-B_{2}-\lambda_{x, k+1} r x_{k}^{*}+\lambda_{y, k+1}\left(d+x_{k}^{*}-u_{k}^{*}\right) \\
\lambda_{x, T}=\lambda_{y, T}=0 .
\end{gathered}
$$

Furthermore, $u^{*}$ satisfies

$$
u_{k}^{*}=\min \left\{\max \left\{\frac{-\lambda_{y, k+1} y_{k}^{*}}{B}, 0\right\}, 1\right\} .
$$

The optimality system for our problem consists of the state equations (23), (24), with initial conditions and adjoint equations (26), (27), with final time conditions (28), and with the optimal control characterization (29).

6.2. Numerical Results. As was done in the logistic and Beverton-Holt growth models, the optimality system for the Ricker growth model was solved numerically, using the iterative method described previously. We present the results for various parameters described in Table 3. We varied one parameter from (23)-(24) at a time, while keeping all other parameters in (23)-(24) constant at 1.0. The initial populations are $x_{0}=0.5$ and $y_{0}=1.0$.

As in the previous models, in the Ricker growth model, $B_{2}$ is the balancing coefficient, which affects the importance of decreasing the pest population relative to the goals of maximizing the valuable population and minimizing the cost of applying the control. Figure 8 shows the optimal control as $B_{2}$ is varied. As $B_{2}$ increases over the values $0.01,0.1,1.0,10$, the value of the optimal control also increases, showing a positive correlation. Comparing this figure to Figure 5, which shows the control as $B_{2}$ is varied in the Beverton-Holt growth function, shows different control results for these different growth functions. However, both 
TABle 3. Summary of Ricker Spawner-Recruit Parameters

\begin{tabular}{|c|c|c|}
\hline Parameter & Meaning & Value \\
\hline \hline $\mathrm{T}$ & number of time steps & 15 \\
$x_{0}$ & initial valuable population & 0.5 \\
$y_{0}$ & initial pest population & 1 \\
$\mathrm{r}$ & population interaction constant & $0.01,0.1,1,10$ \\
$\mathrm{c}$ & intrinsic growth rate of valuable population & $0.01,0.1,1,10$ \\
$\mathrm{~K}$ & carrying capacity & $0.01,0.1,1,10$ \\
$\mathrm{~d}$ & intrinsic growth rate of pest population & $0.01,0.1,1,10$ \\
$B_{2}$ & balancing coefficient & $0.01,0.1,1,10$ \\
$B_{1}$ & cost coefficient & $0.01,0.1,1,10$ \\
\hline
\end{tabular}

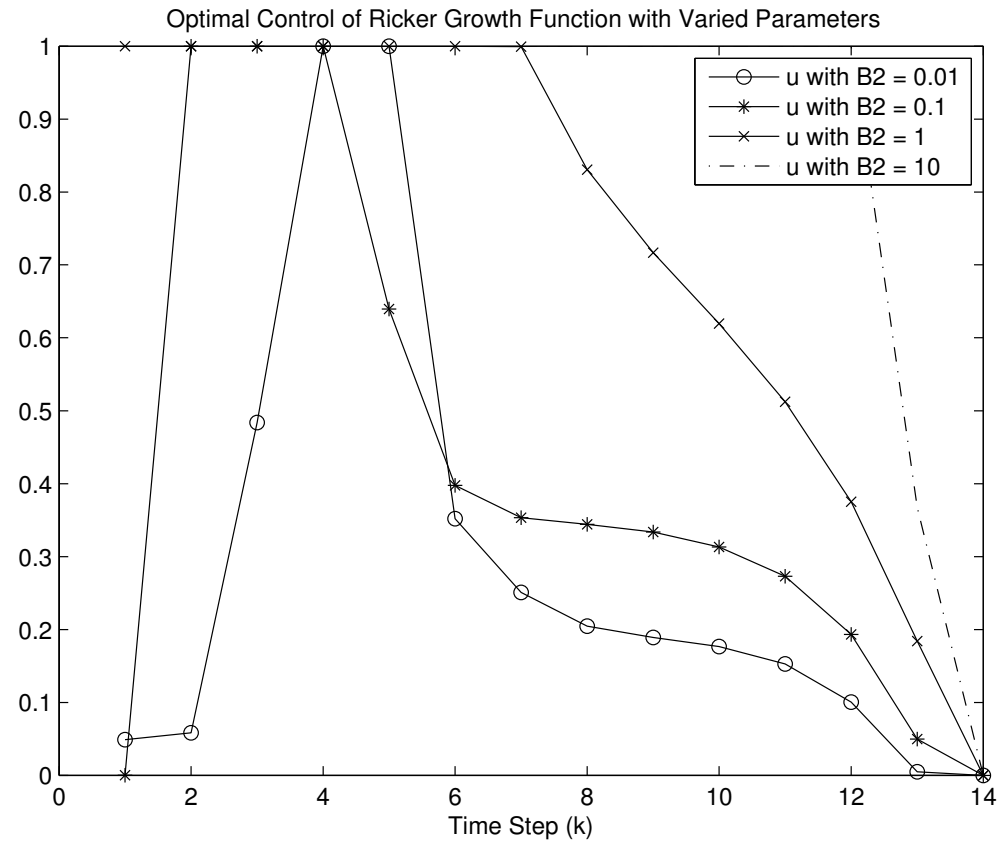

FiguRE 8. Optimal control of Ricker growth function as $B_{2}$ is varied

of these figures represent the same general trend, that as the importance of decreasing the pest population increases, the application of the control also increases.

In the application of the control to the Ricker growth model, $B_{1}$ represents the cost of applying the control. Greater values of $B_{1}$ correspond to a greater cost of applying the control. Figure 9 shows the optimal control as this cost coefficient is varied. As $B_{1}$ increases 


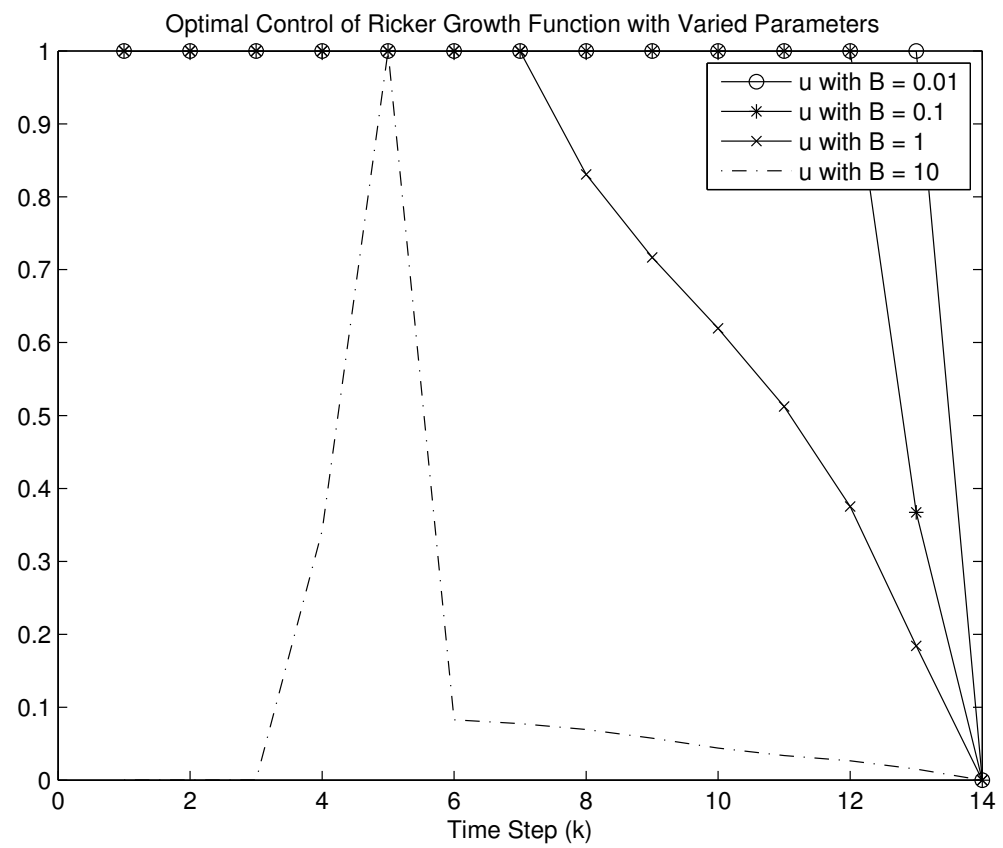

FiguRE 9. Optimal control of Ricker growth function as $B_{1}$ is varied

the value of the control decreases over all time steps. These results are expected because, with higher costs of the control, less control could be obtained to apply to a system.

\section{Comparison of Optimal Control for Different Growth Functions}

The results from the previous sections suggest that the optimal control for systems with different growth functions may respond differently to similar variations in parameters. In this section, we will show how the choice of the growth function to describe a population may affect the optimal control results. In each of the following examples, we compare two different growth functions at a time. We show examples of valuable populations for the different growth functions without the control, which exhibit similar growth patterns. Keeping the parameters the same, we then apply the control to these systems and present the control results.

First, we compare the optimal control of the logistic growth function and the Ricker spawner-recruit curve. Figure 7 shows an example of the valuable populations of logistic and Ricker growth functions without the application of the control. These two populations, although represented by different growth functions, seem to exhibit similar growth patterns. With the application of the optimal control, however, the two growth functions no longer show similar patterns. This can be seen in Figure 7. Note that the y-axis in the plot of the Ricker model has a greater range than the one for the logistic model. 


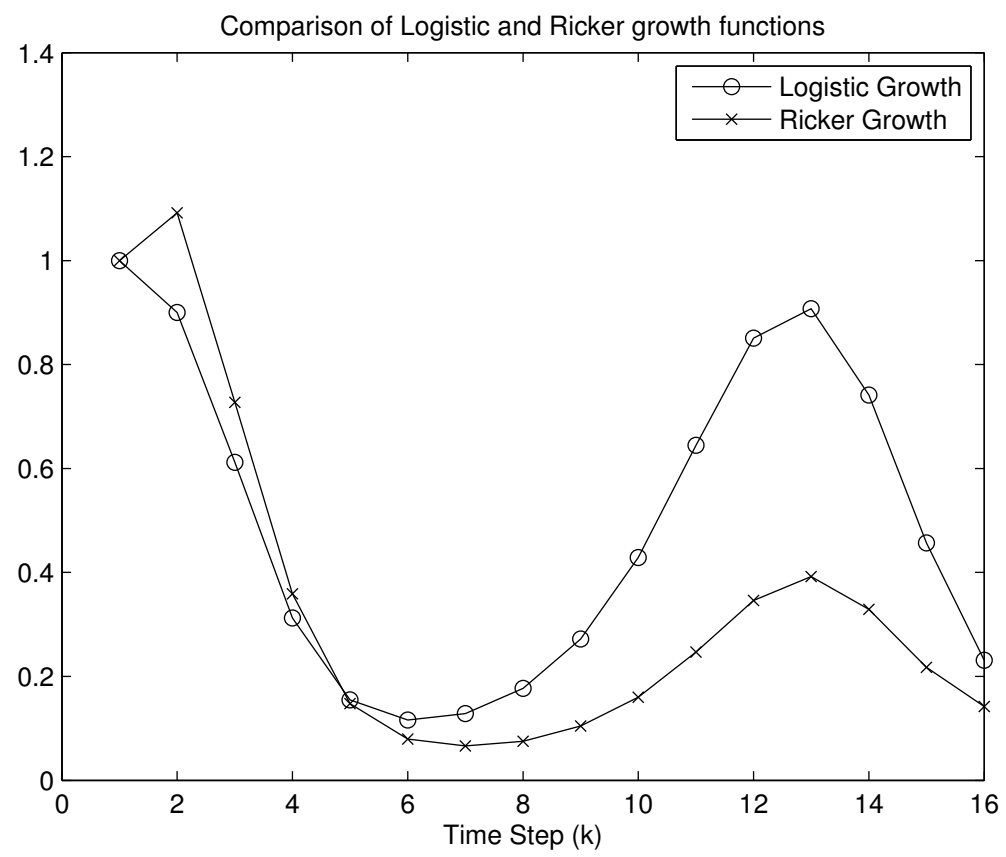

FiguRE 10. Logistic growth function and Ricker spawner-recruit curve without control. For the logistic growth function, $c=1.2, d=0.6, r=$ $0.5, K=1.5$, and $x_{0}=y_{0}=1.0$. For the Ricker function, $c=1.2, d=$ $0.8, r=0.4, K=1.5$, and $x_{0}=y_{0}=1.0$

The difference between these two models can also be seen in the control results, which are shown in Figure 7. In the logistic model, the optimal control is applied with a value of 1.0 for $k=1, \cdots, 11$, and then decreases to 0 by $k=14$. This application of the control allows the valuable population to grow without being overwhelmed by the pest population. In the Ricker model, on the other hand, the control is not applied until after the value of the pest population surpasses the value of the valuable population. Then the control is applied at a value of 1.0 for only one time step before again decreasing to close to 0 . In the Ricker model, less control is needed to decrease the value of the pest population. This may not be ideal from a biological perspective, however, because the control is not applied until the pest population has almost entirely wiped out the valuable population. In the logistic model, although more control is applied for a greater number of time steps, the value of the pest population never exceeds the value of the valuable population.

Next, we compare the optimal control of the logistic growth function and the BevertonHolt growth function. Figure 7 shows an example of the valuable populations for the logistic and Beverton-Holt growth functions without the application of the control. As in the previous example, both of these populations show similar growth patterns. When the optimal control is applied, however, the populations have much different growth patterns, 


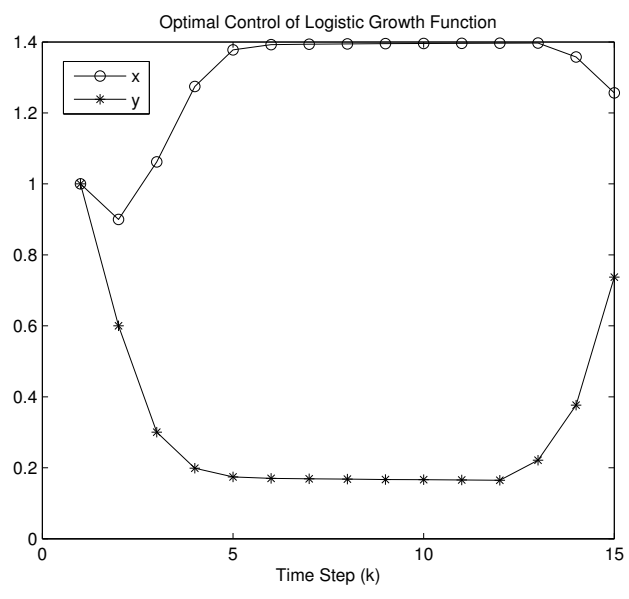

(a) Logistic growth function

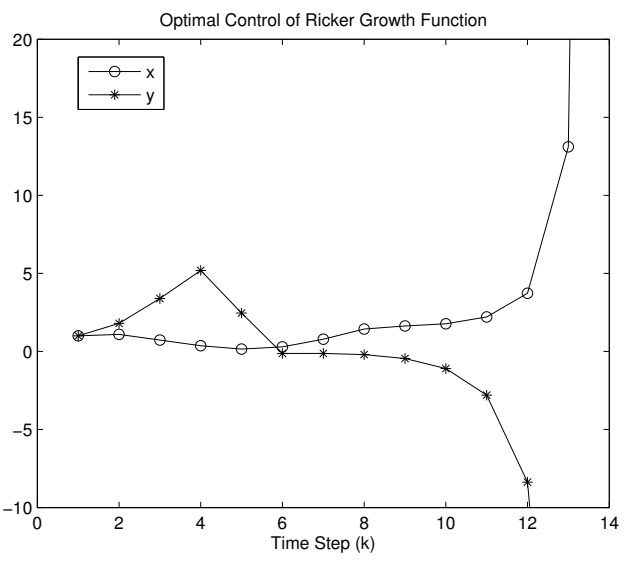

(b) Ricker growth function

FiguRE 11. Valuable and pest populations of the logistic and Ricker growth functions with the application of the control. For both functions, $B_{1}=$ $B_{2}=1.0$.

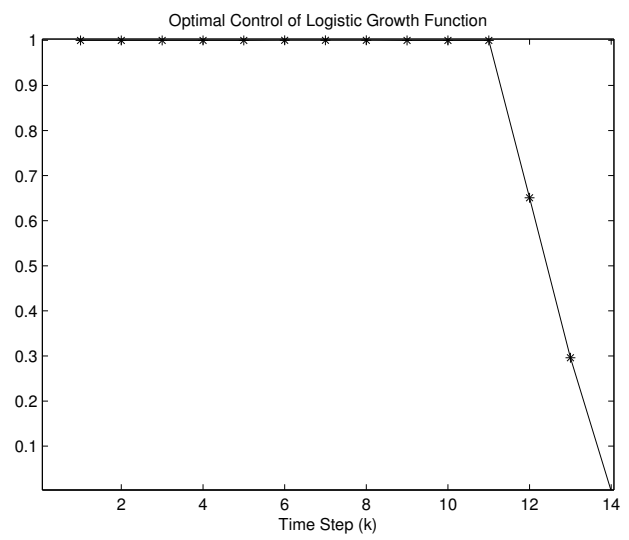

(a) Logistic growth function

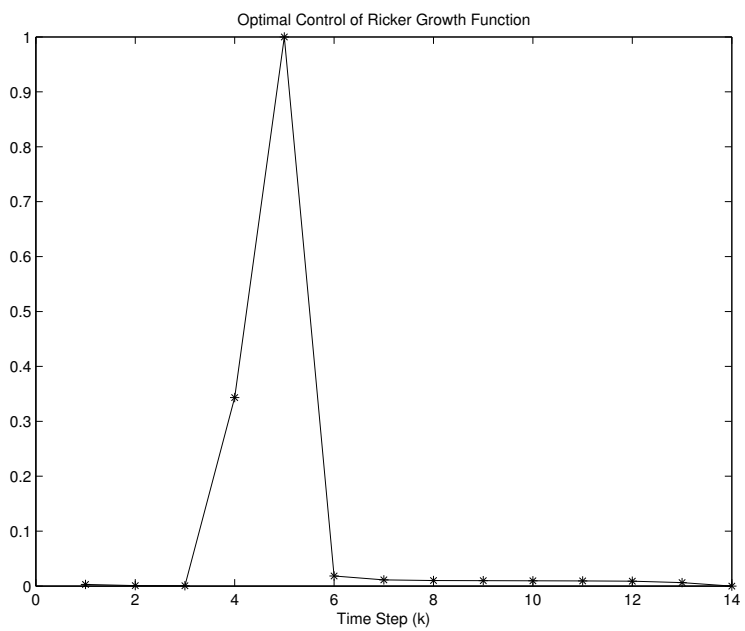

(b) Ricker growth function

Figure 12. Optimal control applied to the logistic and Ricker growth functions in Figure 7. For both functions, $B_{1}=B_{2}=1.0$.

as can be seen in Figure 7. Note that the y-axes are different for the logistic growth model and the Beverton-Holt growth model. 


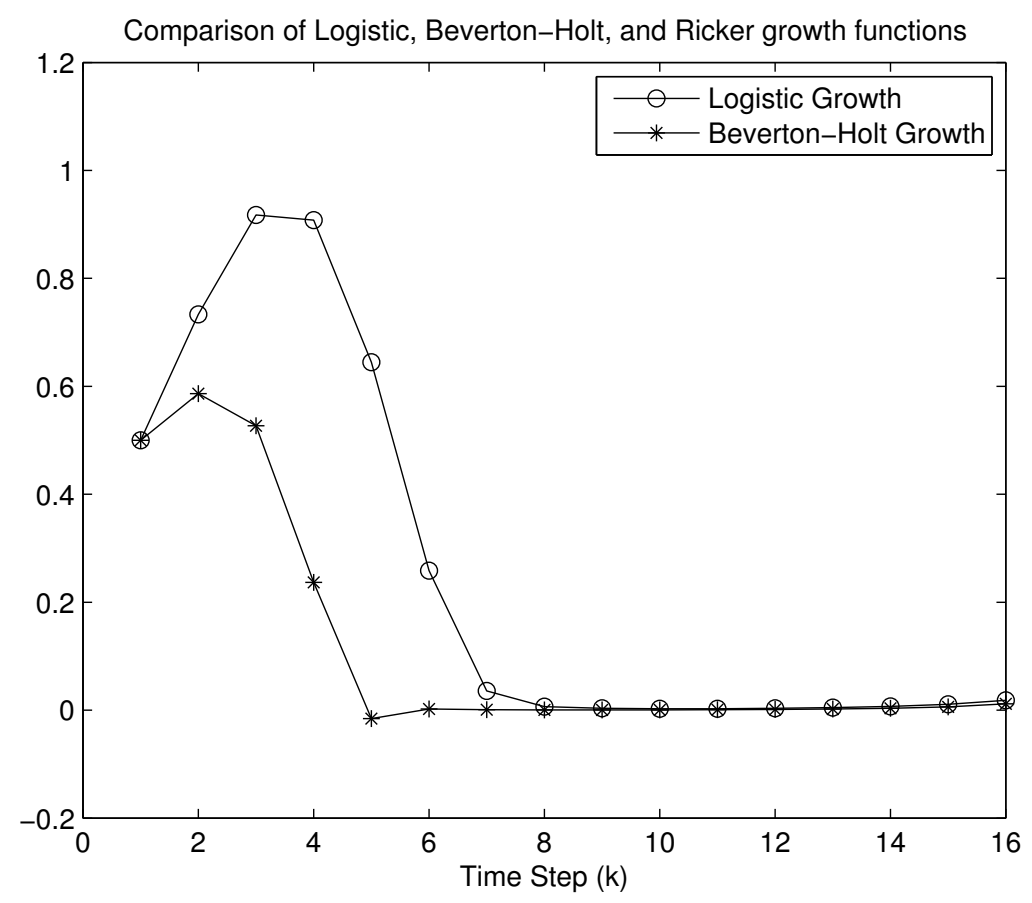

FiguRE 13. Logistic growth function and Beverton-Holt growth function without control. For the logistic growth function, $c=1.0, d=0.8, r=$ $0.2, K=1.5, x_{0}=0.5$, and $y_{0}=1.0$. For the Beverton-Holt growth function, $R=1.1, d=0.8, r=0.9, K=2.0, x_{0}=0.5$, and $y_{0}=1.0$

As in the previous example, the difference between the two models is apparent in the results of the optimal control as well as the effect of the control on the growth of the valuable and pest populations. Similar to the logistic model in the previous example, the control is applied at constant value of about 1.0 for the first 12 time steps, before decreasing to 0. The optimal control in the Beverton-Holt model, on the other hand, has more dynamic application of the control.

\section{Conclusion}

We applied optimal control to discrete time models, which represent the dynamics of two interacting populations, a "pest" population and a "valuable" population, with the goal of minimizing the "pest" population, while maximizing the "valuable" population, and minimizing the cost of applying the control. We first presented the results of applying the control to three different growth functions, which are widely used in biological modeling, the logistic growth function, the Beverton-Holt growth function, and the Ricker spawnerrecruit curve. In each case, we varied parameters to see their effect on the control. We then compared systems, in which these different growth functions exhibited similar growth 


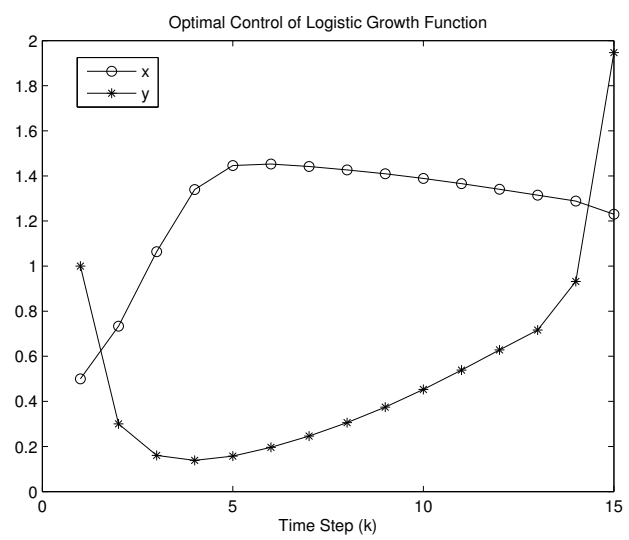

(a) Logistic growth function

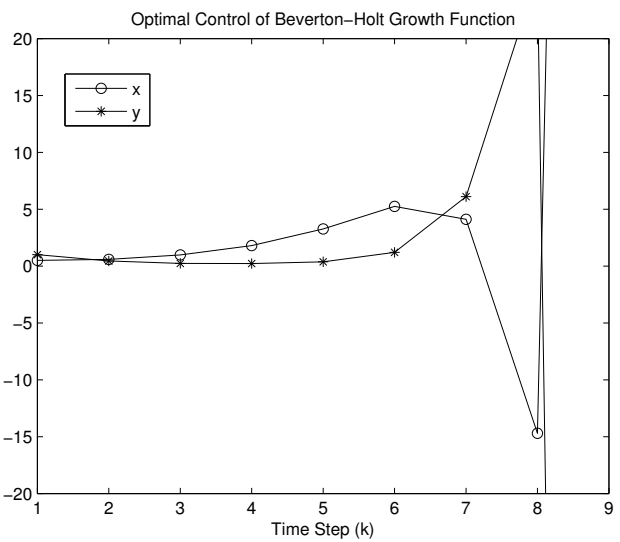

(b) Beverton-Holt growth function

FiguRE 14. Valuable and pest populations of the logistic and BevertonHolt growth functions with the application of the control. For both functions, $B_{1}=B_{2}=1.0$.

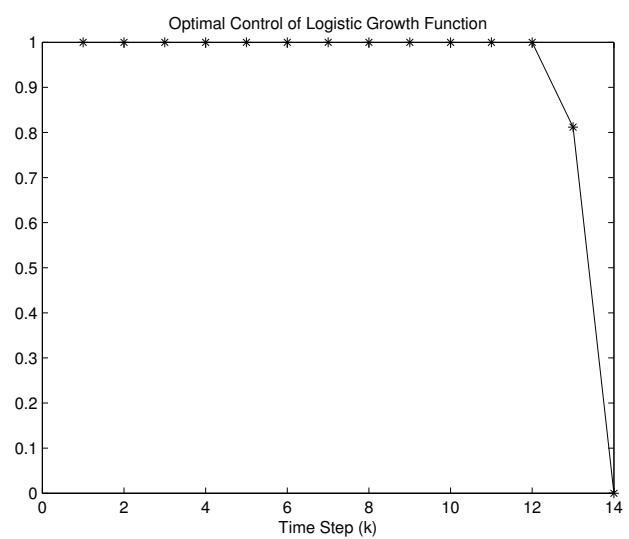

(a) Logistic growth function

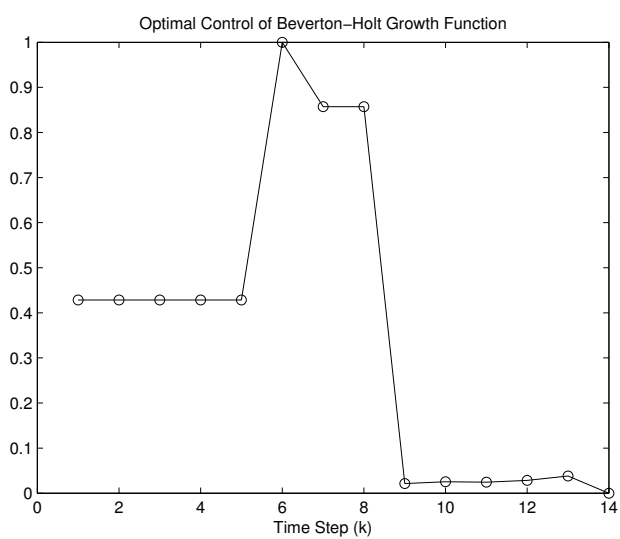

(b) Beverton-Holt growth function

FiguRE 15. Optimal control applied to the logistic and Ricker growth functions in Figure 7. For both functions, $B_{1}=B_{2}=1.0$.

patterns without the application of the control. When applying the optimal control, however, these different models showed different growth patterns and control results. These results suggest that the choice of the growth function to represent the valuable population affects the results of the control, showing that it is important to choose a growth function which accurately describes the dynamics of the biological system. 
8.1. Acknowledgements. This work was completed under the direction of Dr. Suzanne Lenhart and was partially supported by the National Science Foundation. I would also to acknowledge the University of Tennessee Mathematics Honors Program and the advising of Dr. Conrad Plaut.

\section{REFERENCES}

[1] L. J. S. Allen and D. A. Flores and R. K. Ratnayake and J. R. Herbold, Discrete-time deterministic and stochastic models for the spread of rabies, Applied Mathematics and Computation, 132 ( 2002), 132, 271-292.

[2] H. Caswell, Matrix Population Models: Construction, Analysis and Interpretation, Sinauer Press, Sunderland, MA 2001.

[3] W. Ding, L. J. Gross, K. Langston, S. Lenhart and L. S. Real, Rabies in Raccoons: Optimal Control for a Discrete Time Model on a Spatial Grid, J. of Biological Dynamics 1(4), 2007, 379-393.

[4] R. S. Epanchin-Neill and A. Hastings, Controlling established invaders: integrating economics and spread dynamics to determine optimal management, Ecological Letters 13 (4), 2010, 528-541.

[5] B. A. Hawkins and H. V. Cornell, Theoretical Approaches to Biological Control, Cambridge, Cambridge University Press, 1999.

[6] M. Kot, Elements of Mathematical Ecology, Cambridge, MA 2001.

[7] S. Lenhart and J. Workman, Optimal Control Applied to Biological Models, Boca Raton, Chapmal Hall/CRC, 2007.

[8] L.S. Pontryagin, V.G. Boltyanskii, R.V. Gamkrelize, and E.F. Mishchenko, The Mathematical Theory of Optimal Processes, New York, Wiley, 1962.

[9] L. Olson, The economics of terrestrial invasive species: a review of the literature. Agr. Resource Econ. Rev., 35, 2006, 178194. 